Fl uor escent nucl ear track i mages of Ag-act i vat ed phosphate gl ass i r r adi at ed wi th phot ons and heavy charged particles

\begin{tabular}{|l|l|}
\hline 著者 & $\begin{array}{l}\text { Kur obor i Toshi o, Yanagi da Yuka, Kodai ra } \\
\text { Sat oshi, Shi rao Tai chi }\end{array}$ \\
\hline j our nal or \\
publ i cat i on ti t l e & $\begin{array}{l}\text { Nucl ear I nst r ument s and Net hods i n Physi cs } \\
\text { Resear ch, Sect i on A: Accel er at or s, } \\
\text { Spect r onet er s, Det ect or s and Associ at ed } \\
\text { Equi pnent }\end{array}$ \\
\hline vol une & 855 \\
\hline page r ange & $25-31$ \\
\hline year & $2017-$ 05- 21 \\
\hline URL & ht t p: //hdl . handl e. net /2297/47016 \\
\hline
\end{tabular}




\title{
Fluorescent nuclear track images of Ag-activated phosphate glass irradiated with photons and heavy charged particles
}

\author{
Toshio Kurobori $^{1 *}$, Yuka Yanagida ${ }^{2}$, Satoshi Kodaira ${ }^{3}$ and Taichi Shirao ${ }^{4}$ \\ ${ }^{1}$ Graduate School of Natural Science and Technology, Kanazawa University, \\ Kakuma, Kanazawa 920-1192, Japan \\ ${ }^{2}$ Oarai Research Center, Chiyoda Technol Corporation, Oarai-machi, Ibaraki 311- \\ 1313, Japan \\ ${ }^{3}$ National Institute of Radiological Sciences, 4-9-1 Anagawa, Inage-ku, Chiba 263- \\ 8555, Japan \\ ${ }^{4}$ Nikon Instech Co., Ltd., Tanakanishi, Sakyo-ku, Kyoto 606-8221, Japan
}

Keywords: Ag-activated phosphate glass; Fluorescent confocal laser scanning microscopy; Radiophotoluminescence; Heavy charged particles; Threedimensional imaging

In this paper we report about the demonstration of the nuclear track imaging capabilities of Ag-activated phosphate glass. A $375 \mathrm{~nm}$ laser and confocal laser scanning microscopy (CLSM) were respectively used for track excitation and detection. Specifically, the blue and orange radiophotoluminescent (RPL) tracks and dose distributions observed after irradiation with soft X-rays, gamma rays and heavy charged particles (HCPs) are examined. In addition, the origins of the reductions in RPL efficiency for high-dose X-ray irradiation and for irradiation with HCPs with high linear energy transfer (LET) values are investigated via a CLSM and a conventional fluorescent reader and discussed.

${ }^{*}$ Corresponding author. Tel.: +81-76-264-5478; fax: +81-76-234-4132.

*E-mail address: kurobori@staff.kanazawa-u.ac.jp (T. Kurobori). 


\section{Research Highlights $\quad$ NIMA 59687}

$>3 \mathrm{D}$ track images are demonstrated using a confocal laser microscopy.

$>$ Fluorescent track detectors are based on RPL Ag-doped phosphate glass.

$>$ The dose distributions are examined for X-ray, gamma ray and HCP irradiations.

$>$ The origins of the reduction in RPL efficiency are investigated and discussed. 


\section{Introduction}

Silver-activated phosphate glass, the most widely known radiophotoluminescent (RPL) material [1-4], can be used not only in personal, environmental and clinical dosimeters but also in two-dimensional (2D) and three-dimensional (3D) dose imaging detectors [5-7]. These accumulating passive detectors are based on radiation-induced, optically active Agrelated atomic-scale defects; therefore, the ultimate intrinsic spatial resolution of these detectors is several nanometres [7]. All materials that exhibit RPL phenomena, such as the $\mathrm{F}_{2}{ }^{+}(2 \mathrm{Mg})$ centres in $\mathrm{Al}_{2} \mathrm{O}_{3}: \mathrm{C}, \mathrm{Mg}[8]$ and the $\mathrm{Ag}^{0}$ and $\mathrm{Ag}^{2+}$ centres in Ag-activated phosphate glass (hereafter, Ag-glass) [3, 9], have certain prominent features: wide dynamic ranges, high sensitivity, low energy responses and the capability of multiple non-destructive readouts. Other materials that are known to exhibit RPL phenomena include lithium fluoride (LiF) [10] and CsBr:Sm crystals [11].

Fluorescent nuclear track detectors (FNTDs) based on $\mathrm{Al}_{2} \mathrm{O}_{3}: \mathrm{C}, \mathrm{Mg}$ crystals, developed by Landauer, Inc [12], represent one of the most attractive technologies for passive solidstate dosimetry as a novel tool of replacing CR-39 plastic nuclear track detectors (PNTDs) [13]. New technology combining confocal laser scanning microscopy (CLSM) with this novel type of FNTD has been developed for use in neutron detection and dosimetry, proton and heavy ion radiobiology, ion beam cancer therapy and space radiation dosimetry as well as nuclear and particle physics research [14-18]. Recently Kodaira et al. [19] demonstrated the utilisation of Ag-glass as a PNTD. The study opened a new stage to the material for RPL dosimeter as for a nuclear track etch detector. Here we have challenged the utilisation of RPL Ag-glass for a FNTD for the first time to reconstruct 2D and 3D track images of the material irradiated with X-rays, gamma-rays and HCPs. 


\section{NIMA $59687 \quad$ T. Kurobori}

In this paper, the 2D and 3D images determined based on the distributions of radiationinduced $\mathrm{Ag}^{0}$ and $\mathrm{Ag}^{2+}$ defects throughout the entire sample volume in Ag-glass exposed to different X-ray irradiation doses $(1,10$ and $100 \mathrm{~Gy})$ are presented. 3D track imaging results for Ag-glass after irradiation with gamma ray $\left({ }^{137} \mathrm{Cs}\right)$ and heavy charged particles (HCPs) (protons and Fe ions) are examined via CLSM. In addition, the origins of the reductions in RPL efficiency for high-dose X-ray irradiation and for high-LET HCP irradiation are investigated via CLSM and a conventional fluorescent reader and discussed.

\section{Experimental procedures}

\subsection{Samples}

Commercially available Ag-glass plates with dimensions of approximately $8.5 \times 8.5 \times$ $1.5 \mathrm{~mm}^{3}$ were used for all the measurements, including absorption, excitation, emission and fluorescent nuclear track imaging. All facets of the samples were optically polished. The composition of the material by weight was the same as that of FD-7 (AGC Techno Glass Co., Ltd., Japan), i.e., $31.55 \% \mathrm{P}, 51.16 \% \mathrm{O}, 6.12 \% \mathrm{Al}, 11.00 \% \mathrm{Na}$ and $0.17 \% \mathrm{Ag}$. The mass density, photon effective atomic number and refractive index of this material are $2.61 \mathrm{~g} / \mathrm{cm}^{3}$, 12.57 and 1.52 [6], respectively. After irradiation, all of the samples were preheated to 100 ${ }^{\circ} \mathrm{C}$ for $10 \mathrm{~min}$ to promote and complete the 'build-up' kinetics [9, 20].

\subsection{X-rays, gamma rays and heavy charged particles}

Soft X-ray irradiations were performed using an X-ray unit with a copper target operating at a voltage of $30 \mathrm{kV}$ and a current of $20 \mathrm{~mA}$. The $\mathrm{X}$-ray radiation covered the narrow spectral interval of the part of the soft X-ray region by adding filtration, i.e., quasi-monochromatic 


\section{NIMA $59687 \quad$ T. Kurobori}

radiation, strongly peaked at approximately $8.04 \mathrm{keV}\left(\mathrm{K} \alpha_{1}\right.$ and $\mathrm{K} \alpha_{2}$ lines $)$ with a minor contribution at $8.9 \mathrm{keV}\left(\mathrm{K} \beta_{1}\right.$ line $)$. The absorbed doses delivered to the samples were 1,10 and $100 \mathrm{~Gy}$. The samples were placed at a distance from the tube of approximately $10 \mathrm{~cm}$.

Gamma irradiations were performed at a distance of $50 \mathrm{~cm}$ from a ${ }^{137} \mathrm{Cs}(662 \mathrm{keV})$ source that delivered a dose of 1 Gy at the Oarai Research Center, Chiyoda Technol Corporation, Japan.

5 Gy irradiations with a variety of high-energy heavy ions (detailed in Section 3.) were performed at the Heavy Ion Medical Accelerator (HIMAC) of the National Institute of Radiological Sciences (NIRS) in Chiba, Japan. X-ray beams were incident perpendicular to the sample surface $\left(8.5 \times 8.5 \mathrm{~mm}^{2}\right.$ plane $)$.

\subsection{Steady-state optical properties of the samples}

The steady-state optical absorption spectra were determined at room temperature (RT) using a Hitachi U-3900H spectrophotometer with a $1 \mathrm{~nm}$ step. All excitation (EXC) and emission (RPL) spectra were recorded at RT using a Hitachi F-2500 fluorescence spectrophotometer. The ionoluminescent spectra were collected with a resolution of $2.5 \mathrm{~nm}$ and detected by a R928 photomultiplier (PMT) (Hamamatsu Photonics, Japan). All the spectra were corrected for the instrumental calibration. In this work, the blue and orange RPL signals were acquired through a long-pass filter passing all wavelengths longer than 400 nm (\#84-754, Edmund Optics, USA).

\subsection{Fluorescent nuclear track image acquisition}

Preliminary investigations of fluorescent nuclear track imaging were performed after the 


\section{NIMA $59687 \quad$ T. Kurobori}

exposure of the samples to various types of radiation, including X-rays, gamma-rays and HCPs, at RT. A Nikon C2+ CLSM instrument (along with its software, NIS Elements version 4.40) was used to acquire and plot fluorescent 2D and 3D images of the Ag-glass samples after irradiation with photons and HCPs. In this work, instead of the $405 \mathrm{~nm}$ line which is the shortest available excitation wavelength for a normal C2+ CLSM instrument [7], $375 \mathrm{~nm}$ light emitted from a continuous wave $(\mathrm{CW})$ laser diode with a nominal output power of 16 $\mathrm{mW}$ (less than $1 \mathrm{~mW}$ at the sample) (CUBE 375-16C, Coherent, Inc., USA) was used to excite the $\mathrm{Ag}^{2+}$ and $\mathrm{Ag}^{0}$ absorption bands in the Ag-glass.

For the C2+ CLSM instrument, three objective lenses (Nikon Plan Apo) with different magnifications, numerical apertures (NA) and working distances (WD in mm) as well as different immersion liquids were used: the applied configurations were $10 \times / 0.45 \mathrm{NA} / 4.0 \mathrm{WD}$, $40 \times / 1.25 \mathrm{NA} / 0.16 \mathrm{WD} /$ water immersion (refractive index $\mathrm{n}=1.33$ ), and $60 \times / 1.40 \mathrm{NA} / 0.13 \mathrm{WD} /$ oil immersion $(\mathrm{n}=1.52)$. The microscope resolution used in this work are defined by the system's point spread function (PSF) and the lateral size of the PSF (FWHM) $d_{x y}$ and the axial size $d_{z}$ are estimated for an excitation wavelength given $375 \mathrm{~nm}$ : $d_{x y}=508 \mathrm{~nm}, d_{z}=1850 \mathrm{~nm}$ for the $10 \times / 0.45$ NA objective lens, $d_{x y}=183 \mathrm{~nm}, d_{z}=319 \mathrm{~nm}$ for the $40 \times / 1.25 \mathrm{NA}$ lens and $\mathrm{d}_{\mathrm{xy}}=163 \mathrm{~nm}, \mathrm{~d}_{\mathrm{z}}=126 \mathrm{~nm}$ for the $60 \times / 1.40 \mathrm{NA}$ lens, respectively. The blue RPL signals attributed to $\mathrm{Ag}^{0}$ centres and the orange RPL signals attributed to $\mathrm{Ag}^{2+}$ centres were acquired using a band-pass filter $(450 \pm 25 \mathrm{~nm})$ and a long-pass filter passing all wavelengths longer than $561 \mathrm{~nm}$, respectively. Moreover, a pinhole of $40 \mu \mathrm{m}$ in diameter was installed in front of the detector. Such a confocal detection scheme allowed for high spatial and depth resolution under the condition of one-photon absorption at $375 \mathrm{~nm}$, even when areas of the sample adjacent to the focal spot of the excitation light produce fluorescence. 


\section{Results and discussion}

Fig. 1 presents the steady-state optical absorption spectra, including background (BG, non-irradiated) spectra (a) as well as excitation (EXC) (b) and emission (RPL) (c) spectra of Ag-glass samples after X-ray irradiation at an energy of $8 \mathrm{keV}$. The samples were exposed to doses of 1, 10 and 100 Gy to examine and compare the effects of the dose on the optical curves. The observed absorption features are attributed to the superposition of a number of individual absorption bands in the range from 200 to $700 \mathrm{~nm}$, corresponding to single $\mathrm{Ag}^{+}$ ions, neutral $\mathrm{Ag}$ atoms, charged $\mathrm{Ag}_{\mathrm{n}}{ }^{\mathrm{m}+}$ clusters, neutral $\mathrm{Ag}_{\mathrm{n}}$ clusters, metal $\mathrm{Ag}$ nanoparticles and phosphorus-oxygen-hole centres, as previously reported [9, 20, 21].

Among them, two bands are dominant: one is the $310 \mathrm{~nm}$ absorption band attributed to the hole-trapped $\mathrm{Ag}^{2+}$ centres, and the other is the $340 \mathrm{~nm}$ band attributed to the electrontrapped $\mathrm{Ag}^{0}$ centres [1-3]. Exciting the former band results in emission at $630 \mathrm{~nm}$ (the socalled 'orange RPL' signal) with a slow, $2300 \mathrm{~ns}$ decay time, whereas exciting the latter band results in emission at $450 \mathrm{~nm}$ (the so-called 'blue RPL' signal) with a fast, $4.5 \mathrm{~ns}$ decay time [9]. Note that blue and orange RPL signals are simultaneously emitted at excitation wavelengths ranging from 310 to $380 \mathrm{~nm}$ because of the overlap between the broad excitation bands of the $\mathrm{Ag}^{0}$ and $\mathrm{Ag}^{2+}$ centres, as shown in Fig. 1(b), although the intensity ratio between the orange and blue RPL signals $\left(=\mathrm{I}_{\mathrm{o}} / \mathrm{I}_{\mathrm{b}}\right)$ varies.

As shown in Fig. 1(a), the individual absorption bands at 310 and $340 \mathrm{~nm}$ gradually increase in intensity while retaining their shapes as the absorbed dose increases, up to the maximum investigated dose of $100 \mathrm{~Gy}$. However, the intensities of the corresponding EXC and RPL spectra drastically decrease at a dose of 100 Gy, as shown in Figs. 1(b) and (c). The 


\section{NIMA $59687 \quad$ T. Kurobori}

overall results, including the data presented on the Chiyoda Technol Corporation website [22], indicate good linearity of the dose response from $<20 \mu \mathrm{Gy}$ to $10 \mathrm{~Gy}$ for an Ag-glass dosimeter.

Figs. 2(a) and (b) show 2D and 3D fluorescent noise ('background') images acquired after X-ray irradiation at a dose of $1 \mathrm{~Gy}$, which may be primarily attributed to the shot noise of the PMT used as the detector. The noise distribution generally appears at a low intensity level for the maximum bit depth of 12 bits (4096 colours) used here. To eliminate this noise distribution, the look-up table (LUT) provided by the CLSM software was effectively used. These 3D images of X-ray-irradiated samples, with a $1.27 \times 1.27 \mathrm{~mm}^{2}$ field of view and a total of 136 image layers separated by increment of $7.56 \mu \mathrm{m}$ at depths ranging from 0 to 1.02 $\mathrm{mm}$ below the sample surface, were acquired and reconstructed using the $10 \times / 0.45 \mathrm{NA} / 4.0 \mathrm{WD}$ objective lens. With regard to the noise images, one must also consider the following factors: Greilich et al. [18] have reported, based on measurements performed using single-crystalline $\mathrm{Al}_{2} \mathrm{O}_{3}: \mathrm{C}, \mathrm{Mg}$-based FNTDs and a commercial CLSM instrument, that the 'background' varies significantly both within a single crystal and from crystal to crystal because of dust, auto-fluorescent particles and imperfections on the FNTD surface as well as vibrations and electromagnetic interference.

As shown in Fig. 3, 3D fluorescent images of Ag-glass samples irradiated with X-ray doses of 1 (a), 10 (b) and 100 Gy (c) were reconstructed using a CLSM instrument. The images were acquired using two different optical filters: a band-pass filter with a range of $450 \pm 25 \mathrm{~nm}$ for the acquisition of the blue RPL signals and a filter that transmits wavelengths longer than $561 \mathrm{~nm}$ for the acquisition of the orange RPL signals. The bright blue ('blue RPL') and pink ('orange RPL') tracks represent the RPL signals from X-rayinduced luminescent $\mathrm{Ag}^{0}$ and $\mathrm{Ag}^{2+}$ defects, respectively. The noise distribution, as shown in 


\section{NIMA $59687 \quad$ T. Kurobori}

Fig. 2(b), was completely eliminated from each of these images. Note that these 3D images were scanned from the back surface (with a starting point of $z=0 \mu \mathrm{m}$ ) of the X-ray irradiated Ag-glass because the excitation laser beam (at $375 \mathrm{~nm}$ ), the blue RPL signals (at 430 - 480 $\mathrm{nm})$ and the orange RPL signals $(600-650 \mathrm{~nm})$ could not transmit through the impinging surface, especially in the case of the highest irradiation dose of $100 \mathrm{~Gy}$. These reconstructed 3D images, with a field of view of approximately $1.27 \times 1.27 \mathrm{~mm}^{2}$ and a total of 136 layers separated by increments of $7.56 \mu \mathrm{m}$ at depths ranging from 0 to $1.0 \mathrm{~mm}$, were obtained using the $10 \times / 0.45 \mathrm{NA}$ objective lens. As shown in Fig. 3(c), the periodic structures corresponding to the concentrations of the $\mathrm{Ag}^{0}$ and $\mathrm{Ag}^{2+} \mathrm{CCs}$ with pitches ranging from 30 to $50 \mu \mathrm{m}$ (see, Fig. 4(c)), were observed for the first time in the case of X-ray irradiation at a dose of 100 Gy. In order to show the periodic structures clearly, 2D images along the lateral (XY) and axial (XZ and YZ) directions were installed in Fig. 3(d), however, the origins of these structures are now under consideration.

Figs. 4(a), (b) and (c) present the depth dose distribution profiles of 3D images corresponding to Figs. 3(a), (b) and (c), respectively. Fixed wavelengths of 420 and $590 \mathrm{~nm}$ were selected to probe the blue and orange RPL signals, respectively, using 32 channelarrayed-PMT detectors [23] of the CLSM instrument with a resolution of $10 \mathrm{~nm}$. The blue and orange RPL intensity profiles in Fig. 4 were acquired with a limited field of view (in this work, $30 \times 30$ pixels) using ROI (Regions of Interest) functions in Ar (Advanced research) packages of NIS-Elements. It was found that in this case in which we acquire for the whole volume $\left(512 \times 512\right.$ pixels, $\left.1.27 \times 1.27 \times 1.0 \mathrm{~mm}^{3}\right)$, the maximum intensity values of the profiles decreased considerably in comparison with that of the limited region. The blue and orange RPL intensities on each of the layers at different depths (in this work, $7.56 \mu \mathrm{m}$ pitch) are considered to be representative of the corresponding concentrations of radiation- 


\section{NIMA 59687 T. Kurobori}

induced $\mathrm{Ag}^{0}$ and $\mathrm{Ag}^{2+} \mathrm{CCs}$. Therefore, these results suggest that the blue and orange RPL CCs may be non-uniformly distributed in the sample. Note that the RPL intensity a linear behaviour up to 10 Gy in the CLSM images, but at the used highest doses of 100 Gy saturation effects take place. Therefore, the RPL intensity profiles in Fig. 4(c) do not reflect the accurate maximum values, especially at the vicinity of the surface within the sample.

In addition, it was found that a dense radiation-induced defect distribution formed within the region of the sample extending from the surface on which the X-rays were incident to approximately $350 \mu \mathrm{m}$ in depth. The X-ray attenuation length, at which the RPL intensity decreases to a factor of $\mathrm{e}^{-1}=0.368$ with respect to its peak value at the surface, in phosphate glass at the utilised X-ray energy of $8 \mathrm{keV}$ has been calculated to be $340 \mu \mathrm{m} \mathrm{[24]} \mathrm{in} \mathrm{good}$ accordance with the experimental results obtained with 1 and 10 Gy irradiation doses.

Yasuda et al. [25] have noted that the reduction in RPL efficiency in an Ag-glass detector for high-dose ${ }^{60} \mathrm{Co}$ gamma-ray irradiation in the range of $1-60 \mathrm{~Gy}\left(\mathrm{H}_{2} \mathrm{O}\right)$ can be primarily attributed to the attenuation of the UV radiation at $337 \mathrm{~nm}$ caused by the colouring of the material rather than to the saturation of the trapping or recombination centres in the dosimeter. Moreover, the reduction in RPL efficiency with an increasing gamma-ray dose was reported to begin at approximately $6 \mathrm{~Gy}$.

In our case examined via CLSM, although similar behaviour was observed in Ag-glass irradiated with X-rays, the reason for the reduction in RPL intensity is quite different. In our case, the excitation laser beam (at $375 \mathrm{~nm}$ ) and the two RPL signals cannot transmit through the impinging surface, especially in the case of a high X-ray dose of $100 \mathrm{~Gy}$, as shown in Fig. 4(c). Therefore, it is considered that the drastic reduction of the RPL intensity is caused 


\section{NIMA $59687 \quad$ T. Kurobori}

by the saturation of the dense trapping CCs produced at the vicinity of the surface because of the short X-ray attenuation length.

In addition to X-ray irradiation, the observation of the RPL track images in Ag-glass irradiated with gamma-rays and HCPs was also carried out. Fig. 5(a) shows the blue and orange RPL track images acquired from Ag-glass irradiated with a ${ }^{137} \mathrm{Cs}$ gamma-ray with the unrestricted LET $\left(\operatorname{LET}_{\infty}\right)$ value in water of approximately $0.48 \mathrm{keV} / \mu \mathrm{m}$ at a dose of 1 Gy. This 3D image, with a $22.6 \times 22.6 \mu \mathrm{m}^{2}$ field of view and a total of approximately 77 layers separated by increments of $0.527 \mu \mathrm{m}$ at depths ranging from 0 to $40.1 \mu \mathrm{m}$ below the sample surface (with a starting point of $z=0 \mu \mathrm{m}$ ), was reconstructed using the CLSM instrument with the $40 \times / 1.25 \mathrm{NA}$ objective lens configuration.

In addition, Figs. 5(b) and (c) show 3D images acquired via CLSM after irradiation with two types of HCPs, namely, protons $(160 \mathrm{MeV} / \mathrm{u})$ and Fe ions $(500 \mathrm{MeV} / \mathrm{u})$, at a dose of 5 Gy. The LET $\infty$ values in Ag-glass for protons and Fe ions have been calculated to be 1.13 and $5154 \mathrm{keV} / \mu \mathrm{m}$, respectively, using the SRIM-2013 software package [26]. In the case of Fig. 5(b), the 3D image, with a $21.3 \times 21.3 \mu^{2}$ field of view and a total of approximately 179 layers separated by increments of $0.264 \mu \mathrm{m}$ at depths ranging from 0 to $47.0 \mu \mathrm{m}$ below the sample surface, was reconstructed using the $40 \times / 1.25 \mathrm{NA}$ objective lens configuration. Similar image was also acquired for an Fe-ion-irradiated Ag-glass sample. In the case of Fig. 5 (c), the 3D image, with a $21.2 \times 21.2 \mu \mathrm{m}^{2}$ field of view and a total of approximately 346 layers separated by increments of $0.132 \mu \mathrm{m}$ at depths ranging from 0 to $45.6 \mu \mathrm{m}$ below the sample surface, was reconstructed using the $60 \times / 1.40 \mathrm{NA}$ objective lens configuration. In order to support the evidence of FNTDs, a 2D image for Fe-ion-irradiation was also acquired using the same objective lens configuration, as shown in Fig. 5(d). Each RPL fluorescent image of Fe-ion-tracks was clearly visualized with high spatial resolution of smaller than 


\section{NIMA $59687 \quad$ T. Kurobori}

$\sim 100 \mathrm{~nm}$. It was found that the HCPs with the higher LET value (i.e., the Fe ions) produced a higher density of CCs within the same sample volume.

These 3D images indicate that in the case of proton irradiation, the tracks are far away from each other and the overlapping track area is very small, whereas in the case of Fe-ion irradiation, the mean distance between adjacent tracks decreases and the overlapping track area increases. As a result, the attenuation of the RPL luminescence intensity at the same dose of 5 Gy may be primarily attributed to the so-called 'concentration quenching' of the large number of overlapping tracks rather than to the saturation of the radiation-induced CCs in the sample. This behaviour is explained and supported by the analytical model proposed by Sawakuchi et al. [27] to describe the radial dose distribution (RDD) created by the passage of HCPs through matter.

The projected ranges for protons and Fe ions in Ag-glass can also be calculated using the SRIM-2013 code [26] and range from approximately $81.8 \mathrm{~mm}$ for $160 \mathrm{MeV} / \mathrm{u}{ }^{1} \mathrm{H}$ particles and approximately $79 \mu \mathrm{m}$ for $500 \mathrm{MeV} / \mathrm{u}^{56} \mathrm{Fe}$ particles. In order to observe the Bragg peak, micrometre-scale responses of Ag-glass to focused ionized particle radiation were evaluated by proton beam writing using a $3 \mathrm{MeV}$ focused proton micro-beam. In this case, a $3 \mathrm{MeV}$ proton beam stopped at the estimated projected range $(\sim 75 \mu \mathrm{m}$ depth, figures not shown here).

In addition, irradiation was performed with a variety of HCPs with a dose of $5 \mathrm{~Gy}$ at the NIRS HIMAC, as shown in Fig. 6, which presents a plot of the relative RPL efficiency as a function of the LET $\infty$ value in Ag-glass: samples were exposed to $160 \mathrm{MeV} / \mathrm{u}^{1} \mathrm{H}$ ions, with an LET of $1.13 \mathrm{keV} / \mu \mathrm{m} ; 150 \mathrm{MeV} / \mathrm{u}^{4} \mathrm{He}$ ions, with an LET of $13.4 \mathrm{keV} / \mu \mathrm{m} ; 290 \mathrm{MeV} / \mathrm{u}{ }^{12} \mathrm{C}$ ions, with an LET of $166 \mathrm{keV} / \mu \mathrm{m}, 490 \mathrm{MeV} / \mathrm{u}^{14} \mathrm{Si}$ ions, with an LET of $1133 \mathrm{keV} / \mu \mathrm{m} ; 500$ 


\section{NIMA 59687 T. Kurobori}

$\mathrm{MeV} / \mathrm{u}^{40} \mathrm{Ar}$ ions, with an LET of $2320 \mathrm{keV} / \mu \mathrm{m} ; 500 \mathrm{MeV} / \mathrm{u}{ }^{56} \mathrm{Fe}$ ions, with an LET of 5154 $\mathrm{keV} / \mu \mathrm{m} ; 400 \mathrm{MeV} / \mathrm{u}^{84} \mathrm{Kr}$ ions, with an LET of $10390 \mathrm{keV} / \mu \mathrm{m}$; and $290 \mathrm{MeV} / \mathrm{u}^{132} \mathrm{Xe}$ ions, with an LET of $18150 \mathrm{keV} / \mu \mathrm{m}$.

In this case, the RPL efficiency of the orange RPL signal in the irradiated Ag-glass was measured using a conventional fluorescent reader (FGD-702, Chiyoda Technol Corporation, Japan) through a band-pass filter (with transmission between $600 \mathrm{~nm}$ and $650 \mathrm{~nm}$ ) placed in front of the PMT under stimulation by a pulsed $337 \mathrm{~nm}$ laser. The curve indicates that the RPL efficiency drastically decreased with a $10 \mathrm{keV} / \mu \mathrm{m}$ increase in the LET value and that the luminescence efficiency depends on the LET values of HCPs, which drastically decreases at the high LET region ranging from 13.4 to $18150 \mathrm{keV} / \mu \mathrm{m}$. This result is in good agreement with those of Yasuda and Fujitaka [28] for Ag-glass and Yukihara and McKeever [8] for $\mathrm{Al}_{2} \mathrm{O}_{3}: \mathrm{C}$.

In addition to the 'concentration quenching', the 'fluence saturation' must also be taken into consideration [29]. In general, as increasing in size of radionuclide, then the corresponding fluence becomes lower for the same absorbed dose of $5 \mathrm{~Gy}$. The fluence values in this work are estimated to be $\sim 5.9 \times 10^{9}$ particles $/ \mathrm{cm}^{2}$ with an LET value (water) of $0.53 \mathrm{keV} / \mu \mathrm{m}$ for $160 \mathrm{MeV} / \mathrm{u}^{1} \mathrm{H}$ ions and $\sim 1.6 \times 10^{7}$ particles $/ \mathrm{cm}^{2}$ with an LET value (water) of $196 \mathrm{keV} / \mu \mathrm{m}$ for $500 \mathrm{MeV} / \mathrm{u}^{1} \mathrm{H}$ ions. As a result, the reduction in RPL efficiency for HCP irradiation with higher LET values is closely related to this effect. Further measurements are currently underway.

\section{Conclusions}

The data obtained in this study yielded the following conclusions: 


\section{NIMA $59687 \quad$ T. Kurobori}

(1) For the first time, 2D and 3D track images were successfully recorded and reconstructed from RPL Ag-activated phosphate glasses after irradiation with soft X-rays, gamma-rays (Cs) and HCPs (protons and Fe-ions) under laser stimulation at a wavelength of $375 \mathrm{~nm}$ using a commercial CLSM instrument.

(2) For Ag-glass samples exposed to various X-ray doses (1, 10 and $100 \mathrm{~Gy})$, the 3D track images and the profiles of energy deposition attributed to radiation-induced $\mathrm{Ag}^{0}$ and $\mathrm{Ag}^{2+}$ defects throughout the entire sample volume were examined via CLSM (see, Figs. 3, 4). As a result, data suggested that the drastic reduction of the RPL intensity is caused by the saturation of the dense trapping CCs produced at the vicinity of the surface because of the short X-ray attenuation length. Moreover, the periodic structures with pitches ranging from 30 to $50 \mu \mathrm{m}$ were observed in the case of X-ray irradiation at a dose of $100 \mathrm{~Gy}$, however, the origins of these structures are now under consideration.

(3) The RPL efficiency for HCP irradiations with high LET values ranging from 1.13 to $18150 \mathrm{keV} / \mu \mathrm{m}$ were investigated to clarify the origins of the reductions in RPL efficiency (see, Figs. 5, 6). As a result, it was found that the origins of the RPL efficiency reduction are closely related to the 'concentration quenching' and/or the 'fluence saturation' from the experimental results obtained via a CLSM instrument and a conventional fluorescent reader.

A future study will be conducted to measure 3D fluorescent tracks in Ag-glass by utilising a multiphoton CLSM instrument combined with femtosecond (fs) laser pulses generated by a mode-locked Ti:sapphire laser operating at $700 \mathrm{~nm}$. 
NIMA 59687 T. Kurobori

\section{Acknowledgements}

The authors would like to thank the staff of Chiyoda Technol Corporation for their assistance with the gamma irradiation experiments and for their contributions to the sample preparation. This work was supported by JSPS KAKENHI Grant Numbers JP26289362 and JP15K14290 and partially by the JST Matching Planner Program, Grant Number MP27215667972. The authors would like to thank the paper reviewers for their useful comments and important suggestions. 


\section{References}

[1] R. Yokota, H. Imagawa, Radiophotoluminescent centers in silver-activated phosphate glass. J. Phys. Soc. Jpn 23 (1967) 1038.

[2] J. A. Perry, RPL Dosimetry, Radiophotoluminescence in Health Physics, Medical Science Series (Adam Hilger, Bristol) (1987).

[3] T. Yamamoto, D. Maki, F. Sato, Y. Miyamoto, H. Nanto, T. Iida, The recent investigations of radiophotoluminescence and its application, Radiat. Meas. 46 (2011) 1554.

[4] Y. Miyamoto, H. Nanto, T. Kurobori, Y. Fujimoto, T. Yanagida, J. Ueda, S. Tanabe, T. Yamamoto, RPL in alpha particle irradiated $\mathrm{Ag}^{+}$-doped phosphate glass, Radiat. Meas. 71 (2014) 529.

[5] T. Kurobori, S. Nakamura, A novel disk-type X-ray area imaging detector using radiophotoluminescence in silver-activated phosphate glass, Radiat. Meas. 47 (2012)1009.

[6] T. Kurobori, A. Matoba, Development of accurate two-dimensional dose-imaging detectors using atomic-scale color centers in Ag-activated phosphate glass and LiF thin films, Jpn. J. Appl. Phys. 53 (2014) 02BD14.

[7] T. Kurobori, Y. Yanagida, Y. Q. Chen, A three-dimensional imaging detector based on nano-scale silver-related defects in X- and gamma-ray-irradiated glasses, Jpn. J. Appl. Phys. 55 (2016) 02BC01.

[8] E.G. Yukihara, S.W.S. McKeever, Optically Stimulated Luminescence (Wiley, New York) (2011).

[9] T. Kurobori, W. Zheng, Y. Miyamoto, H. Nanto, T. Yamamoto, The role of silver in the radiophotoluminescent properties in silver-activated phosphate glass and sodium chloride crystal. Opt. Mater. 32 (2010) 1231.

[10] M. Levita, T. Schlesinger, S.S. Friedland, LiF dosimetry based on radiophotoluminescence (RPL), IEEE Trans. Nucl. Sci. 23 (1976) 667.

[11] G. Okada, Y. Fujimoto, H. Tanaka, S. Kasap, T. Yanagida, Sm-doped CsBr crystal as a new radio-photoluminescence (RPL) material, J. Rare Earths, 34 (2016) 769.

[12] G.M. Akselrod, M.S. Akselrod, E.R. Benton, N. Yasuda, A novel $\mathrm{Al}_{2} \mathrm{O}_{3}$ fluorescent nuclear track detector for heavy charged particles and neutrons, Nucl. Instrum. Methods Phys. Res., Sect. B 247 (2006) 295.

[13] B.G. Cartwright, E.K. Sirk, P.B. Price, A nuclear-track-recording polymer of unique sensitivity and resolution, Nucl. Instrum. Meth. 153 (1978) 457.

[14] M.S. Akselrod, R.C. Yoder, G.M. Akselrod, Confocal fluorescent imaging of tracks from heavy charged particles utilizing new $\mathrm{Al}_{2} \mathrm{O}_{3} \mathrm{C}, \mathrm{Mg}$ crystals, Radiat. Prot. Dosim. 119 (2006) 357.

[15] G.J. Sykora, M.S. Akselrod, E.R Benton, N.Yasuda, Spectroscopic properties of novel fluorescent nuclear track detectors for high and low LET charged particles, Radiat. Meas. 43 (2008) 422.

[16] J.A. Bartz, G.J. Sykora, T.H. Underwood, D.N. Nichiporov, G.O. Sawakuchi, M.S. Akselrod, Evaluation of aluminium oxide fluorescent and OSL detectors in proton radiotherapy beams, Radiat. Meas. 46 (2011) 1974.

[17] M.S. Akselrod, G.J. Sykora, Fluorescent nuclear track detector technology - A new way to do passive solid state dosimetry, Radiat. Meas. 46 (2011)1671.

[18] S. Greilich, J.-M. Osinga, M. Niklas, F.M. Lauer, G. Klimpki, F. Bestvater, J.A. Bartz, 
M.S. Akselrod, O. Jäkel, Fluorescent nuclear track detectors as a tool for ion-beam therapy research, Radiat. Meas. 56 (2013) 267.

[19] S. Kodaira, Y. Miyamoto, Y. Koguchi, D. Maki, H. Shinomiya, K. Hanaoka, N. Hasebe, H. Kawashima, M. Kurano, H. Kitamura, Y. Uchihori, K. Ogura, Application of $\mathrm{Ag}^{+}$-doped phosphate glasses as nuclear track etch detectors, Radiat. Meas. 71(2014) 537.

[20] S. Fan, C. Yu, D. He, K. Li, L. Hu, Effect of alkali metal oxides on the properties of radio-photoluminescence glasses, J. Non-Cryst. Solids 357 (2011) 2324.

[21] W. Zheng, T. Kurobori, Assignments and optical properties of X-ray-induced colour centres in blue and orange radioluminescent silver-activated glasses, J. Lumin. 131 (2011) 36.

[22] http://www.c-technol.co.jp/eng/e-p_monitoring

[23] http://www.nikon.com/products/microscope-solutions/lineup/confocal/c2/index.htm

[24] http://henke.lbl.gov/optical_constants/atten2.html

[25] H. Yasuda, M. Takami, T. Ishidoya, Changes in optical transmission caused by gamma ray induced coloring in photoluminescence dosimeter, Health Physics 90 (226) 565.

[26] J.F. Ziegler, M.D. Ziegler, J.P. Biersack, SRIM - The stopping and range of ions in matter (2010), Nucl. Instrum. Methods Phys. Res., Sect. B 268 (2010) 1818.

[27] G.O. Sawakuchi, E. G. Yukihira, S.W.S. McKeever, E.R. Benton, Overlap of heavy charged particle tracks and the change in shape of optically stimulated luminescence curves of $\mathrm{Al}_{2} \mathrm{O}_{3}: \mathrm{C}$ dosimeters, Radiat. Meas. 43 (2008) 194.

[28] H. Yasuda, K. Fujitaka, Responses of TLD $\mathrm{Mg}_{2} \mathrm{SiO}_{4}: \mathrm{Tb}$ and radiophotoluminescent glass to heavy charged particles and space radiation, Radiate. Prot. Dosim. 87 (2000) 115.

[29] M. Piccinini, F. Ambrosini, A. Ampollini, M. Carpanese, L. Picardi, C. Ronsivalle, F. Bonfigli, S. Libera, M. A. Vincenti, R. M. Montereali, Solid state detectors based on point defects in lithium fluoride for advanced proton beam diagnostics, J. Lumin. 156 (2014) 170. 


\section{Figure Captions}

Fig. 1. (Colour online) Steady-state absorption spectra including BG spectra (a) as well as excitation spectra (b) and emission spectra (c) of Ag-glass after X-ray irradiation at various doses.

Fig. 2. 2D (a) and 3D (b) fluorescent noise images acquired from Ag-glass irradiated with $\mathrm{X}$-rays at a dose of $1 \mathrm{~Gy}$.

Fig. 3. (Colour online) Reconstructed 3D images based on the blue and orange RPL tracks in Ag-glass irradiated with X-ray doses of 1 (a), 10 (b) and $100 \mathrm{~Gy}$ (c). Each 3D image was scanned from the back surface (with a starting point of $z=0 \mu \mathrm{m}$ ) of the sample. The $2 \mathrm{D}$ dose distributions in the lateral and axial directions are also shown for the sample irradiated with an X-ray dose of $100 \mathrm{~Gy}(\mathrm{~d})$.

Fig. 4. (Colour online) Blue and orange RPL dose distribution profiles from the top to bottom surfaces within samples exposed to X-ray irradiation at doses of 1 (a), 10 (b) and $100 \mathrm{~Gy}$ (c).

Fig. 5. (Colour online) Blue and orange RPL track image of Ag-glass irradiated with Cs gamma-rays at a dose of 1 Gy, acquired via CLSM (a). Similar 3D RPL tracks of Ag-glass samples irradiated with a dose of 5 Gy of protons (b) and Fe ions (c) and 2D image of Feion-irradiation (d).

Fig. 6. Relative orange RPL efficiencies as a function of the $\mathrm{LET}_{\infty}$ value for the Ag-glass samples irradiated with different HCPs at the same dose of $5 \mathrm{~Gy}$. 
Fig. 1 T.Kurobori

\section{NIMA 59687}
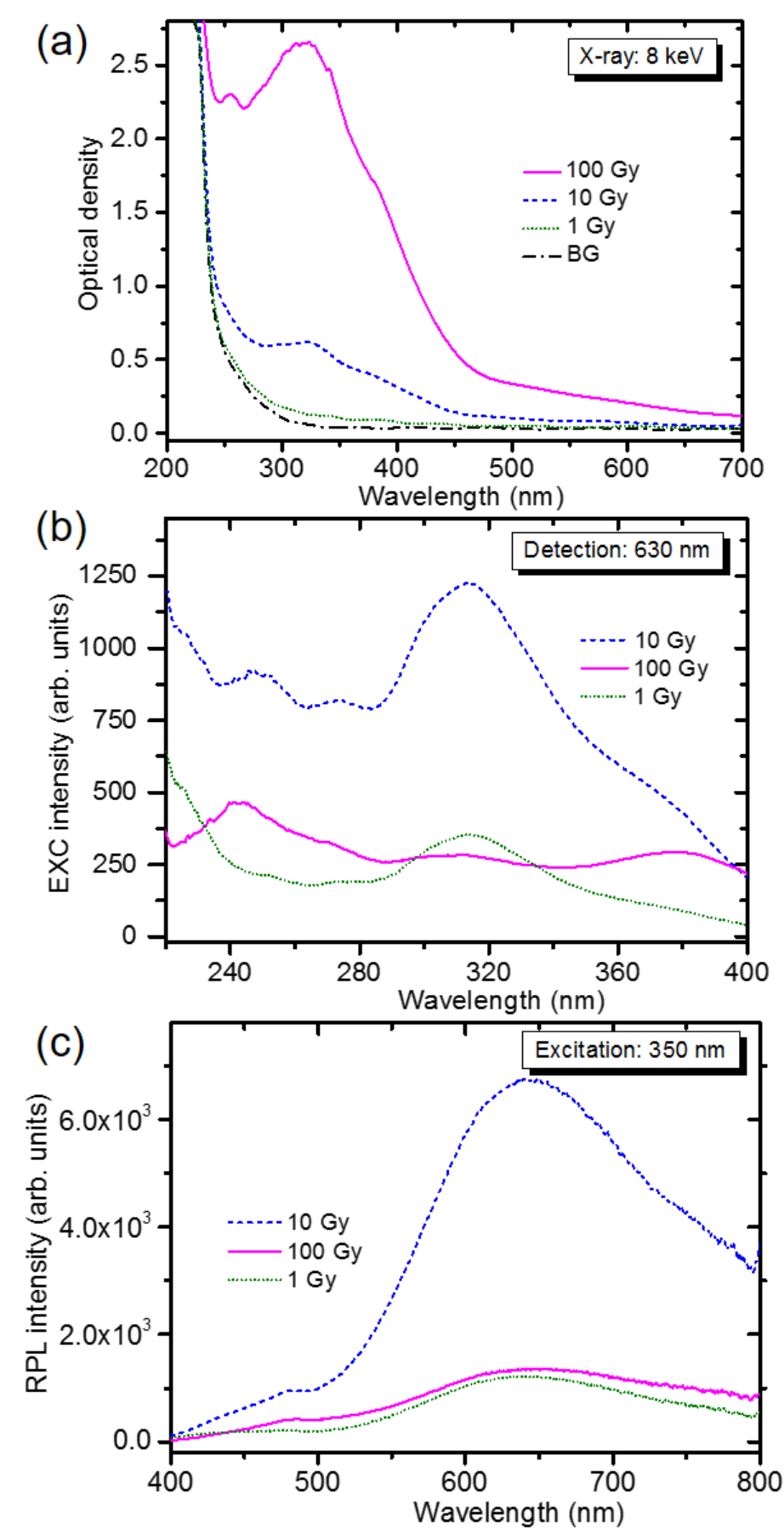
Fig. 2 T.Kurobori

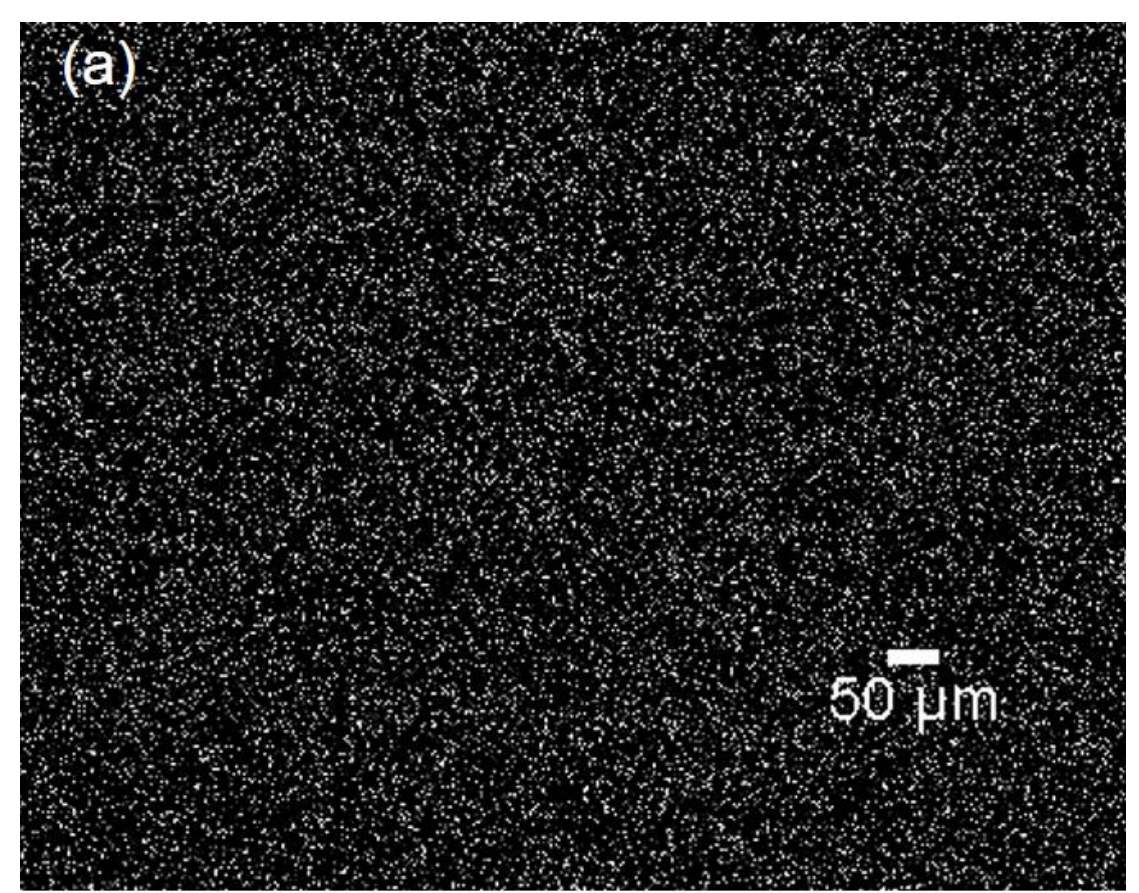

NIMA 59687

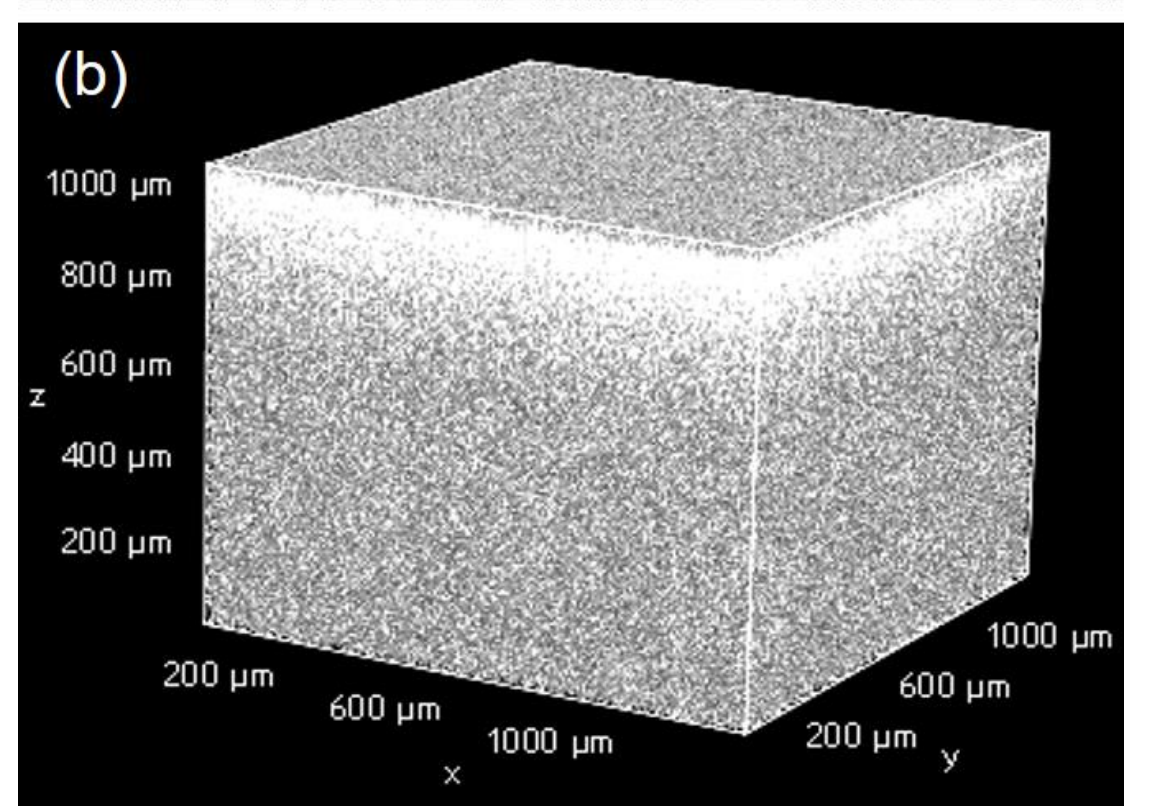


Fig. 3 T.Kurobori

\section{NIMA 59687}
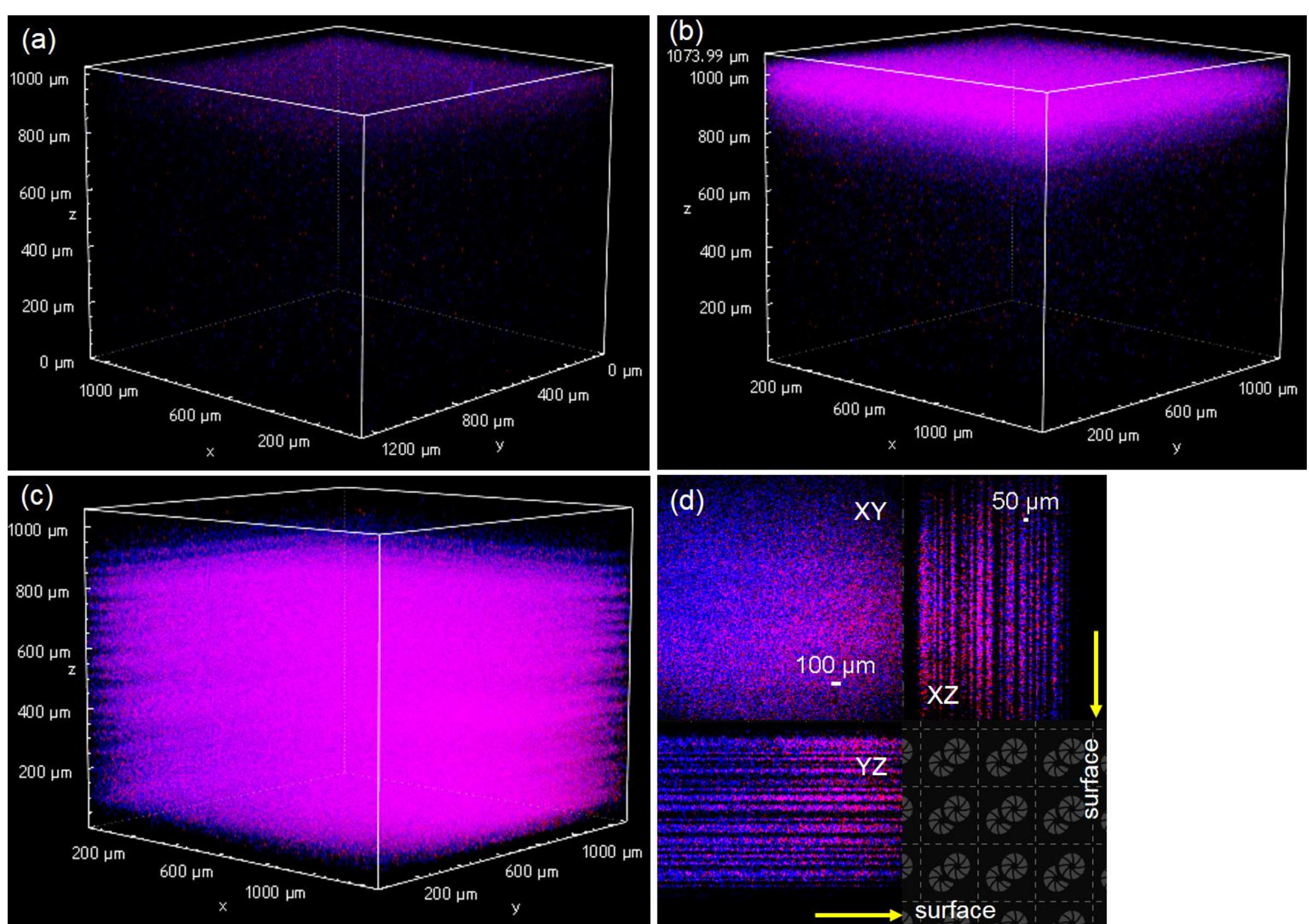

(d)

XY

$50 \mu \mathrm{m}$

$100 \mu \mathrm{m}$

XZ

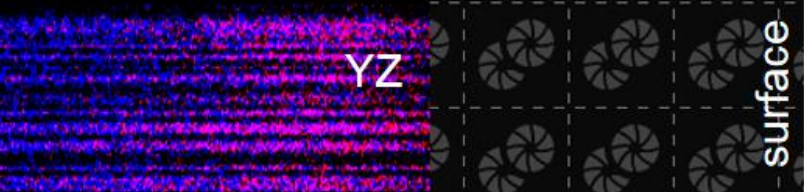


Fig. 4 T.Kurobori

NIMA 59687
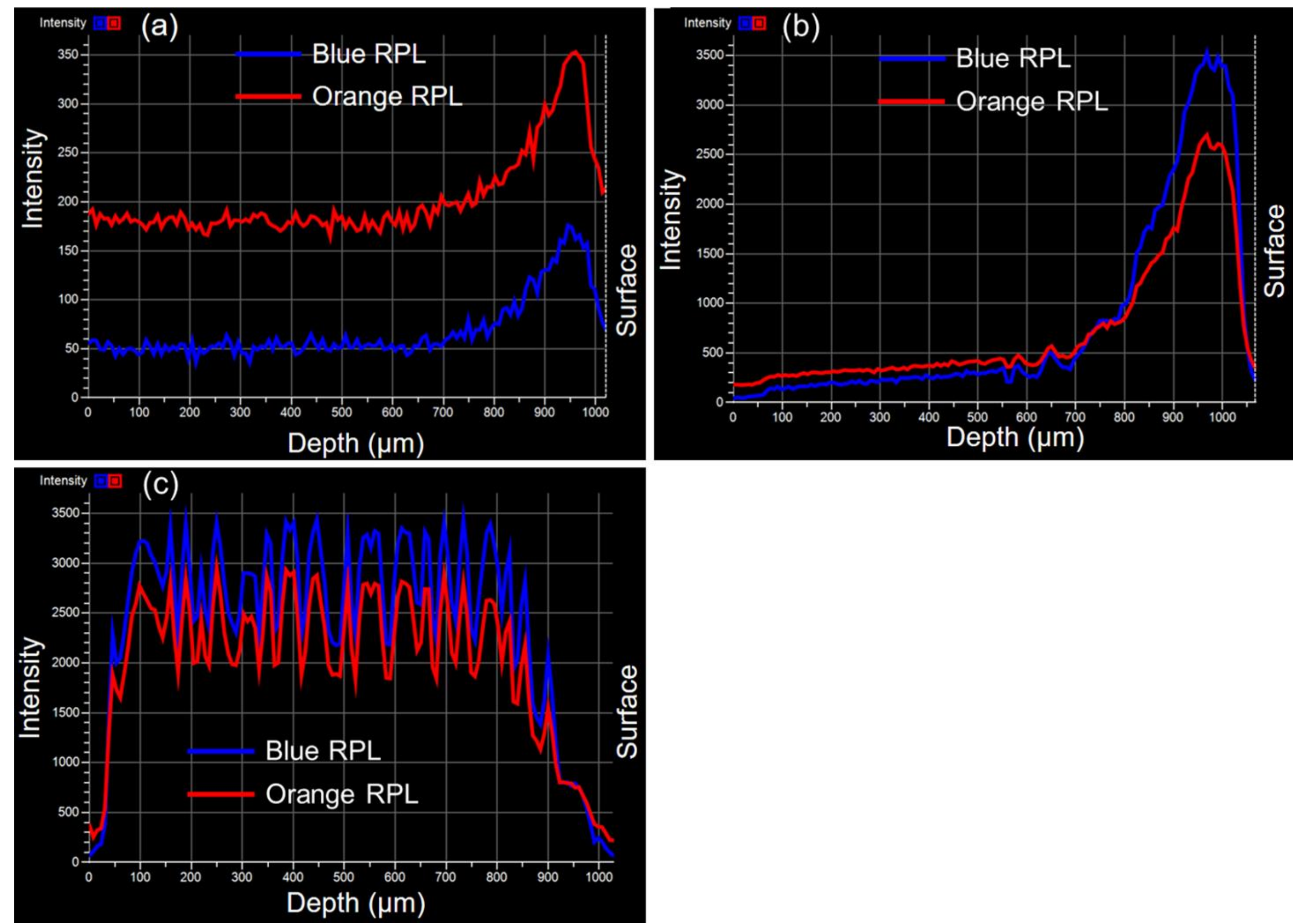

Depth (jm) 
Fig. 5 T.Kurobori

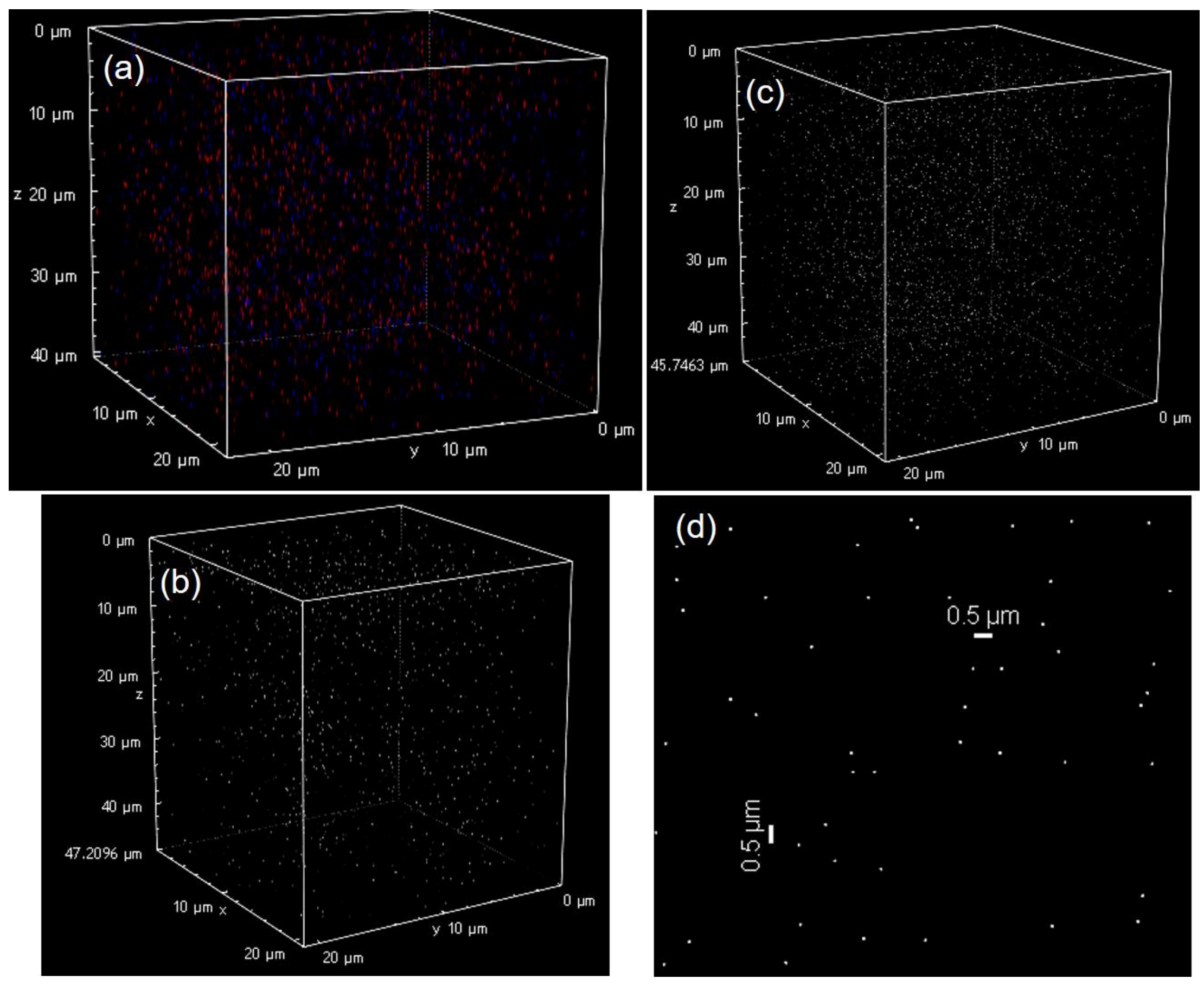


Fig. 6 T.Kurobori

\section{NIMA 59687}

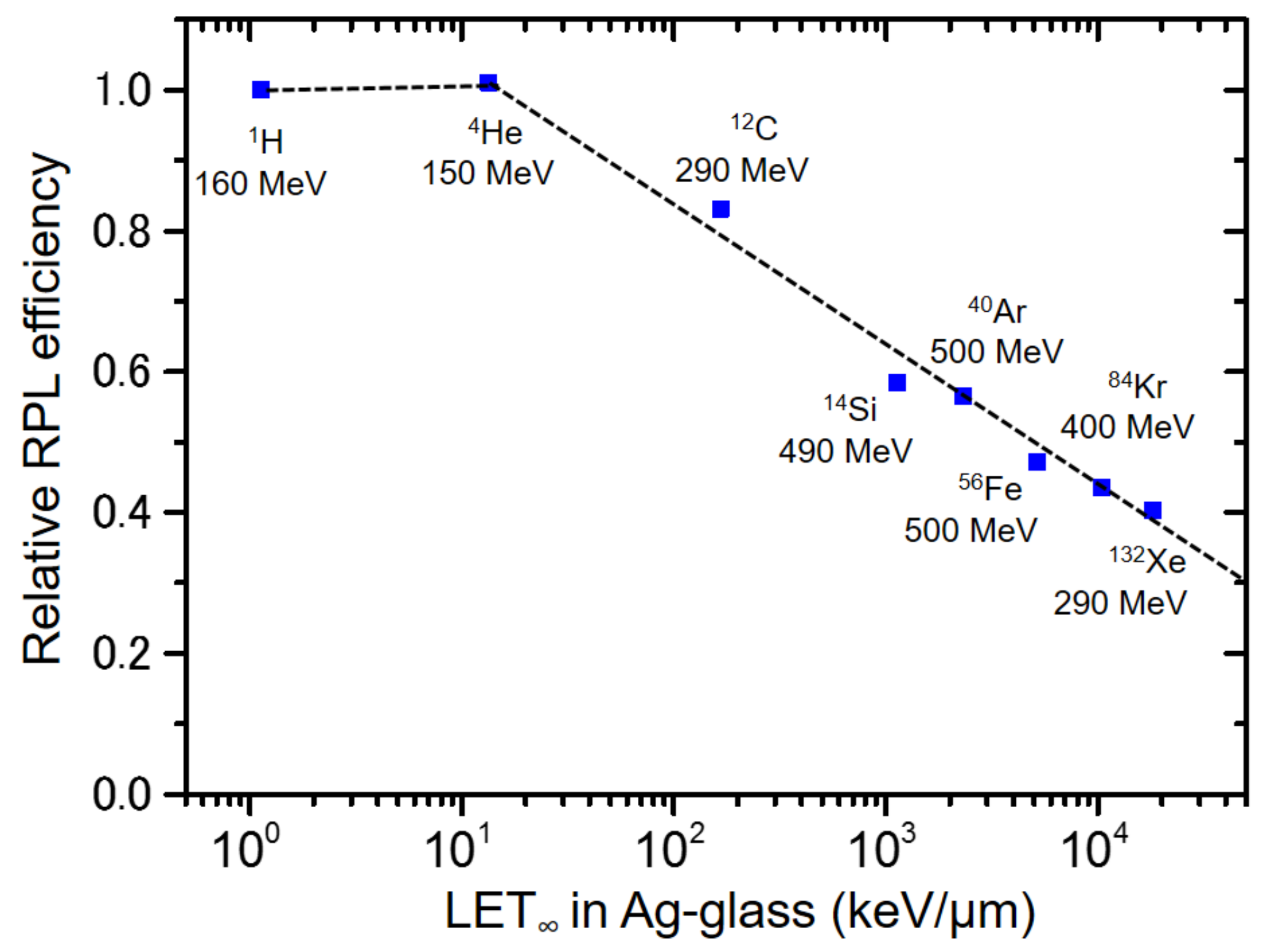

